\title{
VLBA observations of a rare multiple quasar imaging event caused by refraction in the interstellar medium
}

\author{
A. B. Pushkarev ${ }^{1,2,3}$, Y. Y. Kovalev ${ }^{4,1}$, M. L. Lister ${ }^{5}$, T. Hovatta $^{6}$, T. Savolainen ${ }^{1}$, M. F. Aller ${ }^{7}$, H. D. Aller ${ }^{7}$, E. Ros ${ }^{8,9,1}$, \\ J. A. Zensus ${ }^{1}$, J. L. Richards ${ }^{5}$, W. Max-Moerbeck ${ }^{6}$, and A. C. S. Readhead ${ }^{6}$
}

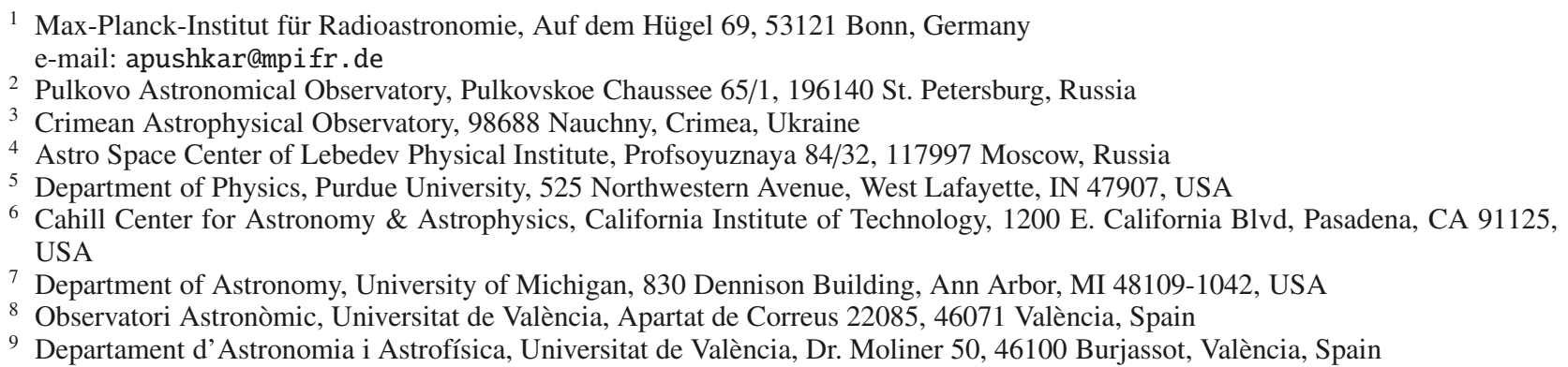

Received 15 March 2013 / Accepted 22 May 2013

\section{ABSTRACT}

\begin{abstract}
Aims. We have investigated highly atypical morphological parsec-scale changes in the flat spectrum extragalactic radio source 2023+335 which are coincident with an extreme scattering event (ESE) seen at radio wavelengths during the first half of 2009. Methods. We used (i) $15.4 \mathrm{GHz}$ Very Long Baseline Array (VLBA) observations of the quasar 2023+335 obtained at 14 epochs between July 2008 and Nov. 2012 as part of the Monitoring Of Jets in Active galactic nuclei with VLBA Experiments (MOJAVE) program; (ii) earlier archival VLBA observations of the source performed at 1.4,2, 8, 15, 22, and $86 \mathrm{GHz}$ to analyze the properties of the proposed turbulent screen toward $2023+335$; and (iii) data sets from the Owens Valley Radio Observatory (OVRO) and University of Michigan Radio Astronomy Observatory (UMRAO) single-dish monitoring programs performed at 15 and $14.5 \mathrm{GHz}$, respectively, to study integrated flux density changes.

Results. We report on the first detection of the theoretically-predicted rare phenomenon of multiple parsec-scale imaging of an active galactic nucleus induced by refractive effects due to localized foreground electron density enhancements, e.g., in an AU-scale plasma lens(es) in the ionized component of the Galactic interstellar medium. We detected multiple imaging in the low galactic latitude $(b=-2.4)$ quasar $2023+335$ from the $15.4 \mathrm{GHz}$ MOJAVE observations when the source was undergoing an ESE. While the parsec-scale jet of the source normally extends along PA -20 , in the 28 May 2009 and 23 July 2009 images a highly significant multi-component pattern of secondary images is stretched out nearly along the constant galactic latitude line with a local $\mathrm{PA} \approx 40^{\circ}$, indicating that the direction of relative motion of the plasma lens is close to orbital. Weaker but still detectable imaging patterns at similar position angles are sporadically manifest at several other epochs. Modeling the ESE that occurred in early 2009 and lasted $\sim 0.14 \mathrm{yr}$, we determined that the foreground screen has a double-lens structure, with proper motion $\left(\sim 6.8 \mathrm{mas} \mathrm{yr}^{-1}\right)$, and angular size ( $\sim 0.27$ mas). We also found that the angular separation between the two brightest sub-images roughly follows a wavelength-squared dependence expected from plasma scattering. Furthermore, by analyzing archival non-simultaneous VLBA observations covering a wide frequency range from 1.4 to $86 \mathrm{GHz}$, we found that the scattered angular size of the VLBI core follows a $v^{-1.89}$ dependence, implying the presence of a turbulent, refractive dominated scattering screen that has a confined structure or is truncated transverse to the line of sight toward $2023+335$.
\end{abstract}

Key words. galaxies: active - galaxies: jets - quasars: individual: 2023+335 - turbulence - scattering

\section{Introduction}

Radio waves are influenced by propagation effects whenever passing through an ionized medium containing free-electron density fluctuations, e.g., in the Earth's ionosphere, the interplanetary medium, or the interstellar medium (ISM). These effects are especially prominent in observations of compact bright sources, such as pulsars, masers, and active galactic nuclei (AGN). They are represented by a wide variety of scattering signatures, including angular broadening, ionospheric or interstellar scintillations, and rare phenomena in AGN radio light curves often referred to as extreme scattering events (ESEs; Fiedler et al. 1987).
The gaseous interstellar medium is characterized by a large Reynolds number, reflecting its highly turbulent nature. The ISM is structured as a hierarchy of clouds that appears selfsimilar over six orders of magnitude in linear scale (Combes 2000). The turbulence in the ISM is mainly driven by supernova explosions, spiral arm instabilities, stellar winds, and cosmic rays (Elmegreen \& Scalo 2004; Lazarian \& Beresnyak 2006). Small-scale turbulent electron density fluctuations can give rise to diffractive scattering, manifested as angular broadening of compact radio sources, while large-scale fluctuations can produce refractive scattering that modulates the overall flux density level, as in the case of scintillations or ESEs. The relative importance of refractive and diffractive scattering depends 
strongly on the form of the spatial power spectrum of electron density turbulence (Goodman \& Narayan 1985; Cordes et al. 1986; Armstrong et al. 1995; Chepurnov \& Lazarian 2010).

The ESE phenomenon, which is characterized by dramatic frequency-dependent changes in source flux density, was first detected during long-term 2.7 and $8.1 \mathrm{GHz}$ monitoring of compact extragalactic radio sources (Fiedler et al. 1987). Because of the simultaneity of the events at different frequencies and speed-of-light travel time arguments, Fiedler et al. (1987) concluded that the events could not be explained by intrinsic variability. It is now broadly accepted (Fiedler et al. 1987, 1994; Romani et al. 1987; Clegg et al. 1988, 1998) that ESEs can be explained by strong scattering, and they occur when ionized material with electron density enhancements and a transverse dimension of $\sim$ AU passes in front of a distant background radio source. Typically, such events last for several weeks to months, and they are quite rare. To date, less than twenty ESEs have been confirmed. What is still unclear about ESEs is the physical nature of the ionized structures (the "lenses") and their relationship to the different phases of the interstellar medium. In this regard, several models have been considered. Clegg et al. (1988, 1998) have proposed that such lenses can be associated with relatively isolated discrete structures in the ISM. Alternatively, Heiles (1997) discussed the possibility of a low level cosmic ray ionization within a neutral structure. Walker \& Wardle (1998) suggested a model of photo-ionized molecular clouds in the Galactic halo. Studying HI absorption before and during an ESE, Lazio et al. (2001) found no changes in equivalent width, maximum optical depth, and velocity at the maximum optical depth, but could not completely rule out the molecular cloud model of Walker \& Wardle (1998), since the observed velocity range covered only about $25 \%$ of the allowed range. The typical electron densities $n_{\mathrm{e}} \gtrsim 10^{2} \mathrm{~cm}^{-3}$ inferred from modeling ESE light curves (Romani et al. 1987; Clegg et al. 1998) lead to pressures $n_{\mathrm{e}} T \gtrsim 10^{6} \mathrm{~K} \mathrm{~cm}^{-3}$ that are $\sim 10^{3}$ times larger than the typical pressure in the ISM (Kulkarni \& Heiles 1987). To overcome this problem, Romani et al. (1987) suggested that ionization fronts and/or cooling instabilities associated with old supernova remnants are the possible sites of the lenses. Moreover, analyzing the high-resolution spectra in the HST archive and deriving ISM pressures along the line of sight, Jenkins \& Tripp (2007) found that a large fraction of the ISM is at the canonical pressure, about $10^{3} \mathrm{~K} \mathrm{~cm}^{-3}$. However, they found a tail in the distribution, extending to pressures $\gtrsim 10^{5} \mathrm{~K} \mathrm{~cm}^{-3}$. Apparently, there are extremely over-pressure regions in the ISM. Their volume filing factor is low, and they may be short-lived, but they clearly exist.

One of the most intriguing predictions of the scattering theory is the possible creation of multiple images of a compact radio source seen through the turbulent screen with refractive dominated properties (Lovelace 1970; Cordes et al. 1986; Rickett \& Coles 1988). Goodman \& Narayan (1985) argued that the image may be broken up into a small number of sub-images, each of which is further fragmented by phase fluctuations on the next smaller scale and so on, thus forming a hierarchical structure similar to a fractal geometry. By investigating refraction in Gaussian plasma lenses as a model of ESE, Clegg et al. (1998) predicted the formation of up to 3 images during periods when the caustic surfaces are formed. Cordes \& Lazio (2001) also reported on a possible creation of multiple imaging by multiple descrete scattering screens. The observable effect of multiple imaging has been detected in the dynamic spectra of pulsars (Cordes \& Wolszczan 1986; Cordes et al. 1986). For AGN, however, there has yet to be a direct detection of the effect. This is largely due to a very low occurrence rate of ESEs ( $\sim 0.01$ event-years per source-year; Fiedler et al. 1994), and the roughly twice as infrequent caustic surface periods when multiple imaging could potentially be detected. The only earlier set of VLBI observations of an AGN during an ESE (the quasar 1741-038) did not reveal any evidence of multiple imaging, presumably because of the insufficient refractive strength of the lens (Lazio et al. 2000).

In this paper, we describe the discovery of multiple imaging of an AGN on parsec scales caused by refraction effects in localized structures with electron density enhancements in the interstellar medium. We analyze a sequence of the $15.4 \mathrm{GHz}$ maps of the quasar 2023+335 obtained within the framework of the Monitoring Of Jets in Active galactic nuclei with VLBA Experiments (MOJAVE) program (Lister et al. 2009a). By modeling the dramatic changes in the total flux density attributed to an extreme scattering event registered by the $15 \mathrm{GHz}$ OVRO observations near 2009.3, we constrain the basic physical characteristics of the intervening plasma lens. Using available multifrequency VLBA observations, we also study the angular broadening of $2023+335$ to derive the properties of the scattering screen toward the source.

Throughout the paper, we use the $\Lambda \mathrm{CDM}$ cosmological model with $H_{0}=71 \mathrm{~km} \mathrm{~s}^{-1} \mathrm{Mpc}^{-1}, \Omega_{\mathrm{m}}=0.27$, and $\Omega_{\Lambda}=0.73$ (Komatsu et al. 2009). All position angles are given in degrees from north through east. The spectral index $\alpha$ is defined according to the convention $S \propto v^{\alpha}$, where $S$ is the flux density and $v$ the observing frequency.

\section{Observations and data reduction}

The extragalactic radio source $2023+335(\mathrm{~J} 2025+3343)$ is a flatspectrum $(\alpha \approx+0.07)$ quasar located at $z=0.22$ (SowardsEmmerd et al. 2003). The quasar is also detected at high energies. It is positionally associated with the X-ray source WGA J2025.1+3342 (Sguera et al. 2004) and with the $\gamma$-ray source 1FGL J2027.6+3335 detected by the Fermi-LAT (Kara et al. 2012).

\subsection{VLBA observations}

$2023+335$ was included into the extension of the statistically complete flux-density-limited MOJAVE sample (Lister et al. 2009a) in July 2008 and has since been monitored at approximately 4 month intervals with the VLBA at an observing frequency of $15.4 \mathrm{GHz}^{1}$. The observations were made in dual circular polarization mode, and recorded with a bit rate of $256 \mathrm{Mbps}$, which was increased to $512 \mathrm{Mbps}$ in the epoch 2008 October 3 and epochs thereafter.

The initial calibration was performed with the NRAO Astronomical Image Processing System (AIPS) (Greisen 2003) following standard data reduction techniques. CLEANing (Högbom 1974), phase and amplitude self-calibration (Jennison 1958; Twiss et al. 1960) were performed in the Caltech Difmap (Shepherd 1997) package. In all cases a point-source model was used as an initial model for the iterative procedure. Final maps were produced by applying natural weighting of the visibility sampling function. The typical uncertainty of the obtained flux densities is $\sim 5 \%$. The absolute calibration of electric vector position angles (EVPAs) is approximately $3^{\circ}$ (Hovatta et al. 2012). The source structure was model-fitted in the visibility $(u, v)$ plane in Difmap using circular and elliptical Gaussian components. For a more detailed discussion of the data reduction and imaging process schemes, see Lister et al. (2009a,b).

http://www . physics. purdue.edu/astro/MOJAVE 
A. B. Pushkarev et al.: Refractive multiple imaging of the quasar $2023+335$

\subsection{Single-dish monitoring}

The quasar $2023+335$ has been frequently monitored with single-dish radio telescopes, including the Owens Valley Radio Observatory (OVRO) 40-m telescope at $15 \mathrm{GHz}$ (Richards et al. 2011). This program, which commenced in late 2007, now includes about 1700 sources, each observed with a nominal twice per week cadence. The OVRO $40 \mathrm{~m}$ uses off-axis dual-beam optics and a cryogenic high electron mobility transistor (HEMT) low-noise amplifier with a $15.0 \mathrm{GHz}$ center frequency and $3 \mathrm{GHz}$ bandwidth. The two sky beams are Dicke-switched using the off-source beam as a reference, and the source is alternated between the two beams in an ON-ON fashion to remove atmospheric and ground contamination. Calibration is achieved using a temperature-stable diode noise source to remove receiver gain drifts, and the flux density scale is derived from observations of 3C 286 assuming the Baars et al. (1977) value of $3.44 \mathrm{Jy}$ at $15.0 \mathrm{GHz}$. The systematic uncertainty in the flux density scale is estimated to be $\sim 5 \%$. Complete details of the reduction and calibration procedure are given by Richards et al. (2011).

Total flux density and linear polarization observations of $2023+335$ were also obtained with the UMRAO Michigan 26-m paraboloid dish as part of the Michigan extragalactic variable source-monitoring program (Aller et al. 1985). The polarimeter consists of dual rotating, linearly polarized feed horns, which are symmetrically placed around the paraboloid's prime focus, and feed a broadband uncooled HEMT amplifier with central frequency $14.5 \mathrm{GHz}$ and bandwidth $1.7 \mathrm{GHz}$. Each daily observation of the target source is an average of 16 measurements over a $40-\mathrm{min}$ time period. The adopted flux density scale is based on Baars et al. (1977). In addition to the observations of this primary standard, observations of nearby secondary flux density calibrators were interleaved with the observations of the target source every 1.5 to $2 \mathrm{~h}$ to verify the stability of the antenna gain and to verify the telescope pointing. The EVPAs were calibrated using a source of known polarized emission mounted at the vertex of the paraboloid. To verify the calibration of the instrumental polarization, selected galactic $\mathrm{H}$ II regions were observed several times each day.

\subsection{Fermi LAT observations}

The Fermi LAT light curve was calculated using an unbinned likelihood analysis (tool gtlike), as implemented in the ScienceTools-v9r27p1 package with P7V6 source event selection. A region of interest of $10^{\circ}$ around the target position was used. All sources within $15^{\circ}$ of the target were included in the likelihood model, and their spectral parameters were frozen to the values obtained from the 2FGL catalog. Other selections and cuts were based on the recommendations from the LAT science team. We used monthly binning and calculated an upper limit if the test statistic (TS) value was 10 or less. During the flaring period (2009.27-2009.65) we used weekly binning to resolve the temporal substructure of the flare.

\section{Arguments for refractive scattering}

\subsection{Serendipitous discovery of unusual parsec-scale structure of the quasar at $15 \mathrm{GHz}$}

In Fig. 1, we present naturally weighted total intensity and linear polarization VLBA images of $2023+335$ at 16 epochs covering a time period of about 11 years. Since the source was not observed in the MOJAVE program prior to 2008, we have reduced the
Table 1. Parameters of the $15 \mathrm{GHz}$ naturally weighted images.

\begin{tabular}{lccccc}
\hline \hline Epoch & $\begin{array}{c}S_{\mathrm{VLBA}} \\
(\mathrm{mJy})\end{array}$ & $\begin{array}{c}S_{\text {base }} \\
\left(\mathrm{mJy} \mathrm{bm}^{-1}\right) \\
(3)\end{array}$ & $\begin{array}{c}P_{\mathrm{VLBA}} \\
(\mathrm{mJy}) \\
(4)\end{array}$ & $\begin{array}{c}P_{\text {base }} \\
\left(\mathrm{mJy} \mathrm{bm}^{-1}\right) \\
(5)\end{array}$ & $\begin{array}{c}\chi \\
\left({ }^{\circ}\right) \\
(6)\end{array}$ \\
\hline $2002-02-15$ & 1715 & 2.5 & 6 & 2.6 & -4 \\
$2005-09-15$ & 2041 & 1.2 & 28 & 1.0 & 9 \\
$2008-07-17$ & 2557 & 0.8 & 52 & 0.8 & 4 \\
$2008-11-26$ & 3228 & 1.5 & 53 & 0.7 & 1 \\
$2009-05-28$ & 4806 & 1.1 & 48 & 0.9 & 60 \\
$2009-07-23$ & 4125 & 0.6 & 16 & 0.9 & 91 \\
$2009-10-27$ & 4952 & 1.0 & 42 & 1.2 & 46 \\
$2009-12-10$ & 5442 & 2.3 & 86 & 2.5 & 43 \\
$2010-02-11$ & 5696 & 1.5 & 120 & 1.2 & 40 \\
$2010-08-06$ & 5294 & 0.9 & 183 & 1.2 & 35 \\
$2010-10-25$ & 3213 & 1.4 & 99 & 1.1 & 29 \\
$2010-12-24$ & 2539 & 1.3 & 44 & 0.8 & 32 \\
$2011-05-21$ & 2884 & 1.2 & 40 & 1.1 & 50 \\
$2011-09-12$ & 2616 & 1.0 & 44 & 0.9 & 28 \\
$2012-07-12$ & 3294 & 1.1 & 68 & 1.1 & 4 \\
$2012-11-11$ & 2960 & 1.0 & 65 & 1.3 & 17 \\
\hline
\end{tabular}

Notes. Columns are as follows: (1) epoch of observations; (2) total VLBA flux density; (3) lowest contour in total intensity image; (4) total VLBA polarized intensity; (5) lowest contour in polarization intensity image; (6) position angle of the electric vector on the sky.

available archival VLBA data at $15 \mathrm{GHz}$ to examine the past structural history of the source. The image parameters are listed in Table 1.

Typically, the milliarcsecond-scale radio morphology of bright, compact AGN jets consists of a one-sided core-jet structure, reflecting strong selection effects and the Doppler boosting of jet emission (e.g., Lister et al. 2009b). The VLBI core component is associated with the apparent origin of AGN jets and commonly appears as the brightest feature in VLBI images. The core is typically unresolved along one or both axes of the restoring beam (Kovalev et al. 2005).

When $2023+335$ was first added to the MOJAVE VLBA imaging program in 2008, the source displayed a classical onesided morphology, with a strong core and a jet extending along position angle $\sim-20^{\circ}$. However, an image taken in May 28, 2009 unexpectedly showed surprising structural changes, with additional bright new emission regions elongated in a direction of position angle $\sim 40^{\circ}$ and $\sim-130^{\circ}$. We have not witnessed this type of behavior in any of the other $~ 300$ AGN jets regularly monitored by the MOJAVE or VLBA $2 \mathrm{~cm}$ survey programs (Kellermann et al. 1998; Zensus et al. 2002; Lister \& Homan 2005; Lister et al. 2009a). Such unusual structural evolution cannot be explained by the standard relativistic jet expansion model (Blandford \& Königl 1979) since it would require bi-directional superluminal expansion - an impossibility according to simple causality arguments. Thus, we rule out the intrinsic variation scenario.

\subsection{Galactic plane sky location}

The first clue to the physical mechanism responsible for these dramatic and quite atypical morphological changes is the fact that the source is at very low galactic latitude $b=-2.37$, suggesting that those structural changes could be caused by some form of propagation effect. Light propagation effects have been seen in other AGN, in the form of scintillation (see e.g., 

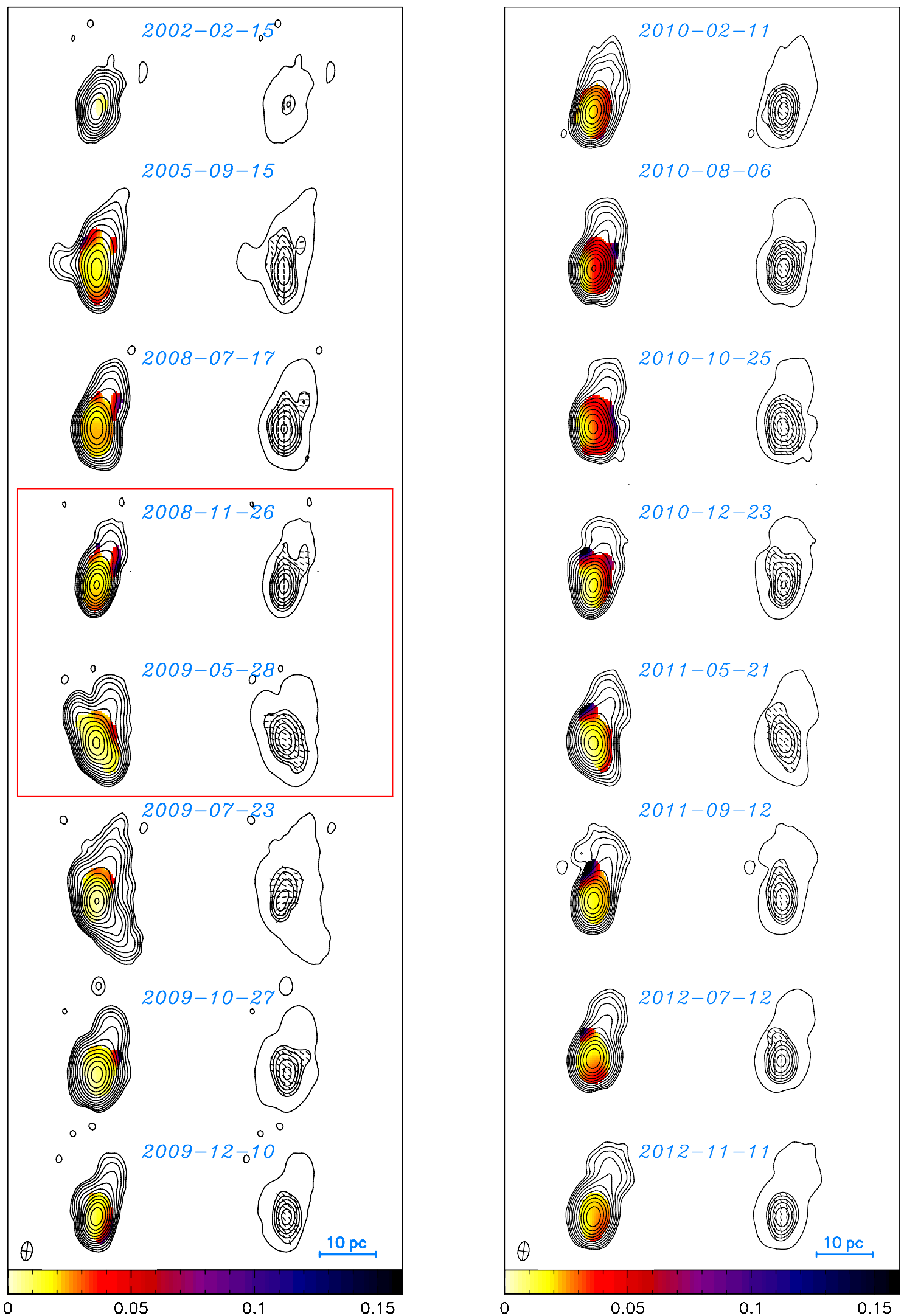

Fig. 1. Sequence of $15.4 \mathrm{GHz}$ VLBA images showing structural changes in the quasar $2023+335$ during a 10.8 yr period. The first two images are non-MOJAVE image from the VLBA archive. The most dramatic parsec-scale variations occurred between 2008.9 and 2009.4 marked by a red box. For each epoch we present two images. The first image is a naturally weighted total intensity map with linear fractional polarization overlaid according to the color wedge. The second image includes the lowest positive total intensity contour from the first image, and linearly polarized intensity contours together with electric polarization vector directions. The crossed ellipse in the lower left represents the FWHM of the restoring beam of $0.96 \times 0.56$ mas at $\mathrm{PA}=-8.2$, which is the median for all restoring beams at the 16 epochs. One milliarcsecond corresponds to about $3.5 \mathrm{pc}$ at the source redshift of $z=0.22$. The image parameters are listed in Table 1 . 


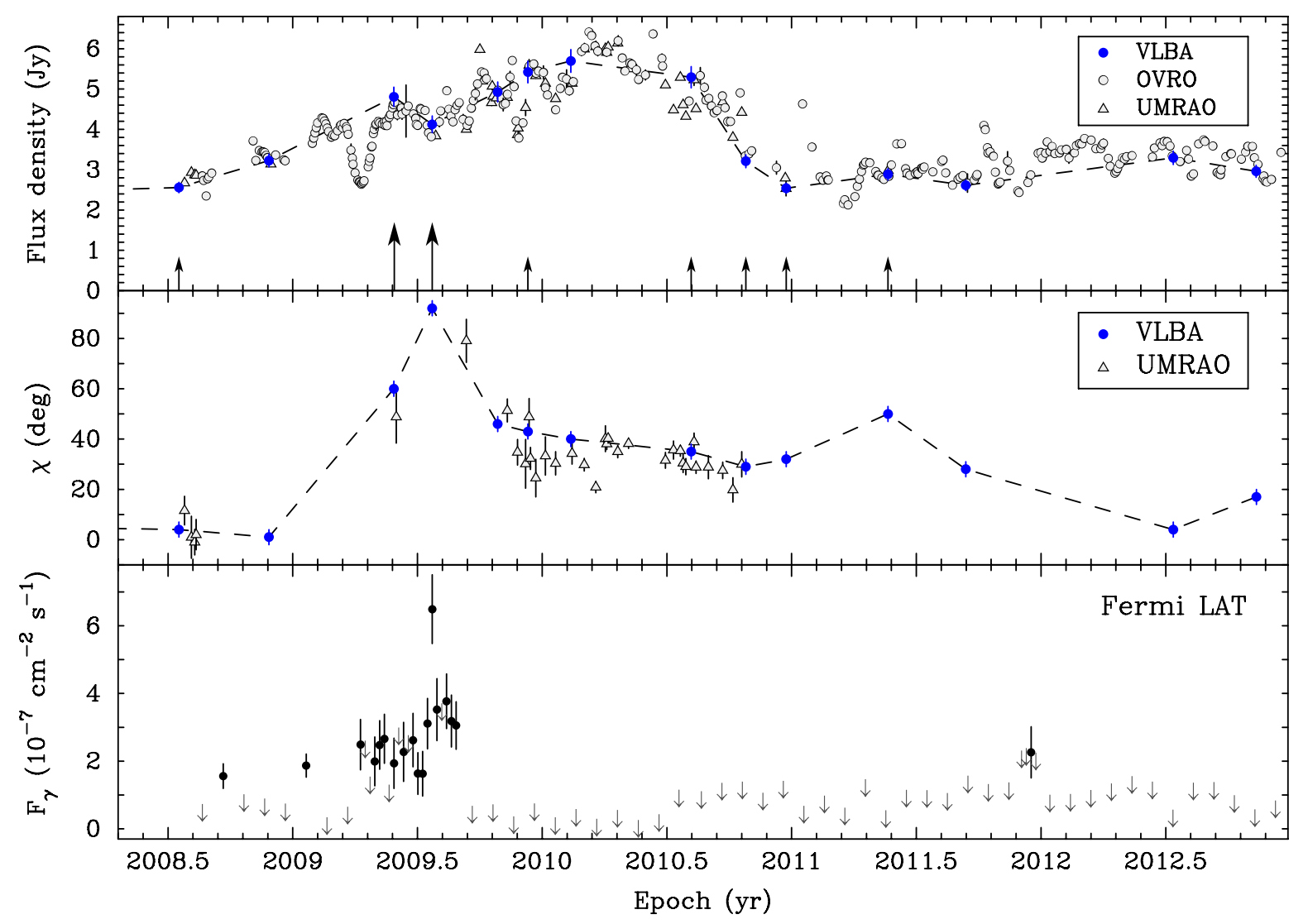

Fig. 2. Top: $15.4 \mathrm{GHz}$ total flux density VLBA (filled circles), $15 \mathrm{GHz}$ OVRO (unfilled circles), and 14.5 GHz UMRAO (unfilled triangles) light curves. The epochs of VLBA observations with signatures of multiple imaging (see Fig. 1) are marked by large (strong manifestation) and small arrows (weaker effect) with distinction made visually from Fig. 1 on the basis of the extension of the refraction-induced parsec-scale core substructure. Middle: electric vector position angle evolution as measured by $15.4 \mathrm{GHz}$ VLBA (filled circles) and single-dish $14.5 \mathrm{GHz}$ UMRAO (unfilled triangles) observations. Bottom: integrated $0.1-200 \mathrm{GeV}$ photon flux measured with the Fermi LAT. Downward arrows indicate $2 \sigma$ upper limits.

Quirrenbach et al. 1989; Jauncey et al. 2000; Dennett-Thorpe \& de Bruyn 2002; Lovell et al. 2008; Savolainen \& Kovalev 2008) or extreme scattering events (Fiedler et al. 1987; Cimò et al. 2002; Senkbeil et al. 2008). The second hint is the galactic longitude $l=73.13$ of the source, which places it behind the highly turbulent Cygnus region (Bochkarev \& Sitnik 1985; Fey et al. 1989a). The line of sight to $2023+335$ passes near the Cygnus loop supernova remnant (Pineault \& Chastenay 1990) that may locally ionize the ISM (Romani et al. 1987).

\subsection{Extreme scattering events}

The final clue to the event can be found in the OVRO light curve (Fig. 2, top panel). This shows that the source was undergoing an ESE around the epoch 2009.3 due to the prominent dip and rise features, as was previously reported by Kara et al. (2012). We can additionally conclude that the refractive strength of the scattering screen must be very high, because all previously known ESEs have been detected at lower radio frequencies, at which the effect is more pronounced. The VLBA observations on May 28, 2009 serendipitously coincided with a special feature of the ESE, the caustic spike, when the secondary images are expected to have the largest angular separations (Fiedler et al. 1987; Romani et al. 1987; Clegg et al. 1998; Lazio et al. 2004). All these facts coherently lead to the conclusion that the new-born structure detected on May 28, 2009, is the result of multiple imaging of the source, induced by refraction in an intervening ISM screen.
We model the physical characteristics of the scattering screen in Sect. 4.

In the current paradigm, an ESE occurs when a localized structure (lens/cloud) of partly-ionized material with electron density enhancements passes in front of a distant background radio source. The significant changes in flux density attributed to ESE are expected when the ratio of the angular size of the lens to the intrinsic angular size of the VLBI core is within a quite narrow range $[1 / n ; n]$, where $n$ is limited by a few. This condition, as well as the turbulence spectrum of a typical screen, determines the expected rate of such events. In addition, the electron density in the screen must be high enough to result in significant scattering that can be detected and recognized as an ESE. ESEs are more prominent at lower frequencies (a few $\mathrm{GHz}$ ), as the refractive power of the lens is greater. This is why most ESEs are detected at $2 \mathrm{GHz}$, some at $6-8 \mathrm{GHz}$, and only one, to our knowledge, at $15 \mathrm{GHz}$ (this paper).

In the case of $2023+335$, which is a galactic plane source seen through the Cygnus region, the probability of observing an ESE is certainly higher than average. For the successful detection of more frequent ESEs, the source should be monitored at lower frequency. Yet even at $15 \mathrm{GHz}$, the OVRO monitoring revealed a clear ESE after only one year of the operation of the program, and has provided evidence for other ESEs in the form of flux density excursions at later epochs (around 2009.9, 2010.05, 2011.2, 2011.7), indicating more complex structure of the scattering screen. Thus, the quasar $2023+335$ is a 


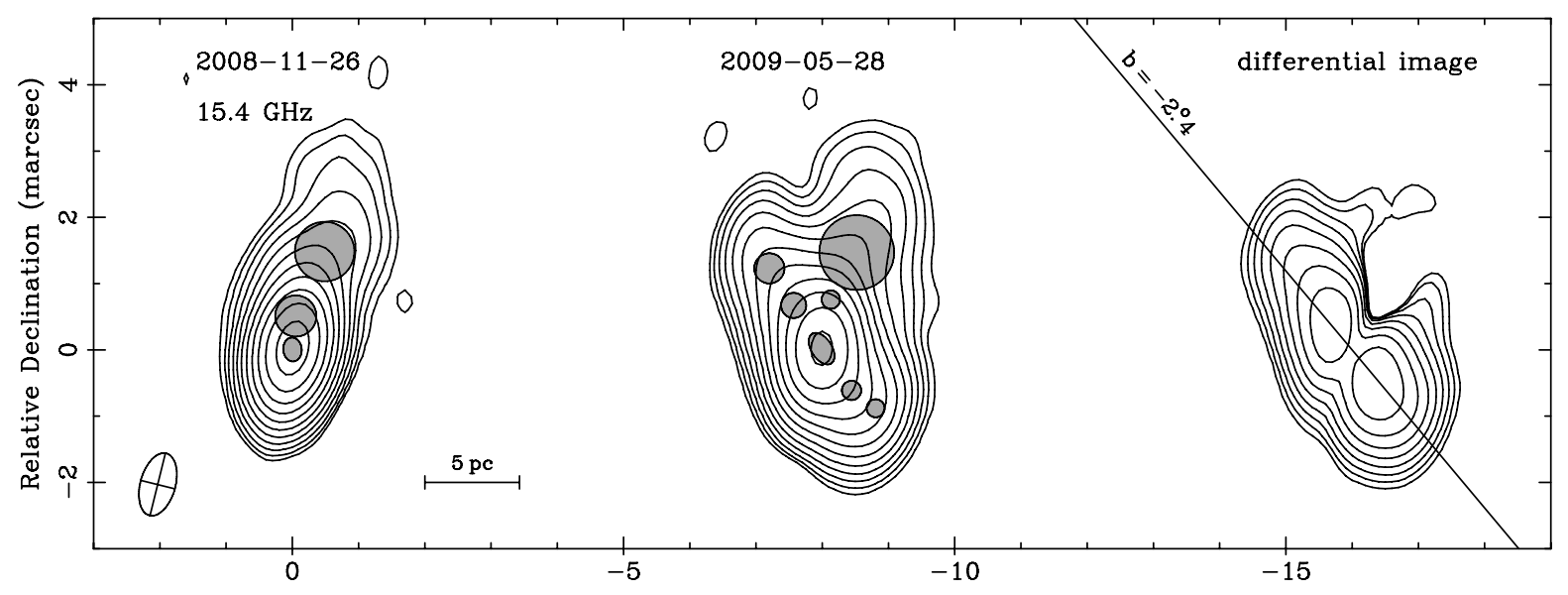

Relative R.A. (marcsec)
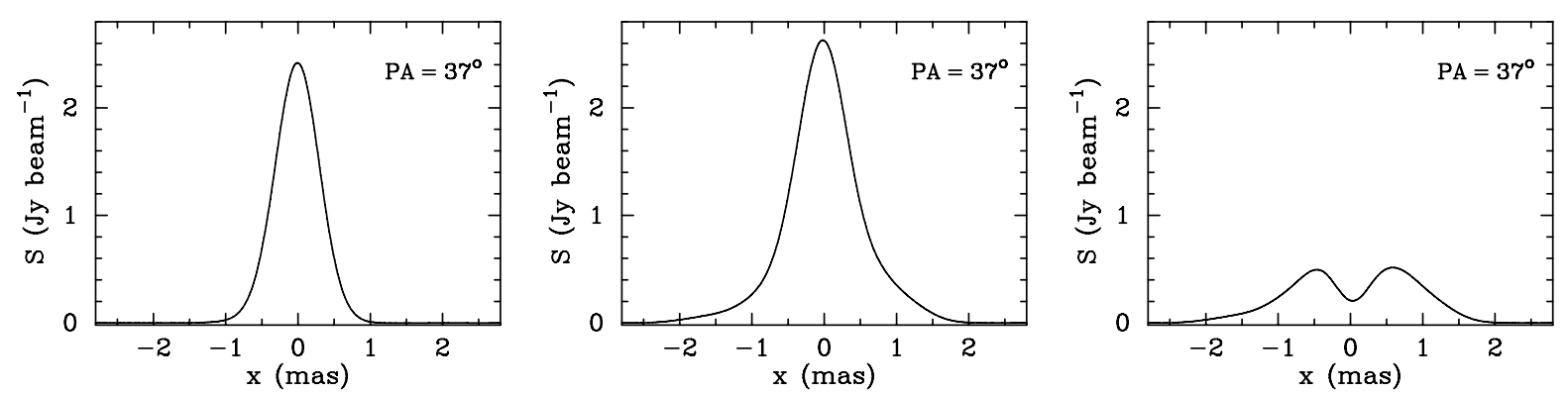

Fig. 3. Extraction of the refraction-induced structure from the naturally weighted $15.4 \mathrm{GHz}$ VLBA images of $2023+335$. The image on 2008 November 26 (top left) with the peak of $2.42 \mathrm{Jy} \mathrm{beam}^{-1}$ shows a typical parsec-scale morphology represented by a bright core and one-sided jet propagating along the $\mathrm{PA} \sim-20^{\circ}$. The image taken on 2009 May 28 (top middle) with a peak flux density of $2.62 \mathrm{Jy}^{\mathrm{b}}$ beam ${ }^{-1}$ shows an unusual brightness distribution, which is a result of multiple imaging of the source. The fitted Gaussian components are superposed on the images as shaded circles/ellipses. The difference image of the two epochs (top right) reveals a quasi-symmetric pattern dominated by two bright components and formed by refraction when the lens edge passes over the source. The pattern of de-magnified secondary images is extended nearly precisely along the line of constant galactic latitude, which is at $\mathrm{PA} \approx 40^{\circ}$ in this region of the sky (full line). All the images were convolved with an identical restoring beam, the FWHM of which is depicted as the crossed ellipse in the lower left corner. One milliarcsecond corresponds to about 3.5 pc. The total intensity profiles (bottom panel) are shown along the $\mathrm{PA}=37^{\circ}$ that connects the peaks of the refractive pattern in the difference image. The angular separation of the peaks is about 1 mas.

promising target for future ESE detections and corresponding studies.

\subsection{Joint analysis of VLBA, OVRO, and UMRAO $15 \mathrm{GHz}$ data supplemented by $\gamma$-ray Fermi results}

To construct an overall picture of what has occurred in the source since $\sim 2008.5$, we analyzed our $15.4 \mathrm{GHz}$ total VLBA flux density measurements, superposed with the more densely sampled OVRO and UMRAO light curves (Fig. 2, top panel). We found good agreement between (i) the VLBA and single-dish flux density measurements, and (ii) the VLBA and UMRAO EVPAs, indicating that virtually all of the emission of $2023+335$ at $15 \mathrm{GHz}$ originates from parsec scales probed by the VLBA. The source was undergoing a flare during the period 2008.5-2010.5, during which it increased its intensity by a factor of $\sim 2$. Most likely, the flare was intrinsic to the source, rather than the result of a focusing effect caused by the ISM. The radio flare is expected to occur when the perturbation moving down the jet crosses the $\tau_{v} \sim 1$ region at a given frequency $v$, the VLBI core. This happens during 2010, when the flux density reaches the maximum of about 6 Jy. Therefore, in 2009 the perturbation is still within the compact VLBI core region, not at significantly larger angular separations, where the new-born emission is detected in May
2009. Moreover, the perturbation is expected to propagate in the direction of the jet $\left(\mathrm{PA} \sim-20^{\circ}\right.$ ), while the observed morphology instead shows the new-born emission appearing at $\mathrm{PA} \sim 40^{\circ}$ and $\mathrm{PA} \sim-130^{\circ}$.

The intrinsic core flare scenario is supported by several observations. First, the flare was accompanied by a rapid $\sim 90^{\circ}$ change in EVPA (Fig. 2, middle panel). This is typically observed when a perturbation crossing the $\tau_{v}=1$ core region at a given frequency, i.e., the VLBI core, changes the regime of synchrotron radiation from being optically thick to optically thin, and the EVPA subsequently returns slowly to the pre-flare value, $\chi \sim 0^{\circ}$, that roughly aligns with the inner jet direction. Second, it is known that the $\gamma$-ray emission is not affected by the ISM, but during the rising part of the radio flare, the source became bright at energies above $100 \mathrm{MeV}$ (Fig. 2, bottom panel), with the $\gamma$-ray peak preceding the $15 \mathrm{GHz}$ one by about 8 months (see also Kara et al. 2012). This is consistent with the established close radio/ $\gamma$-ray connection (e.g., Kovalev et al. 2009; Pushkarev et al. 2010) in the light of a scenario of broadband synchrotron-Compton flares in the base of the jet (e.g., Dermer et al. 2009; Boettcher 2010). Therefore, the flaring in the $15 \mathrm{GHz}$ VLBA core, the apparent jet base, made this most-compact region of the source even more flux-density dominated and thus a region more susceptible to propagation effects in the ISM. The latter are more pronounced for highly compact features. 
A. B. Pushkarev et al.: Refractive multiple imaging of the quasar $2023+335$
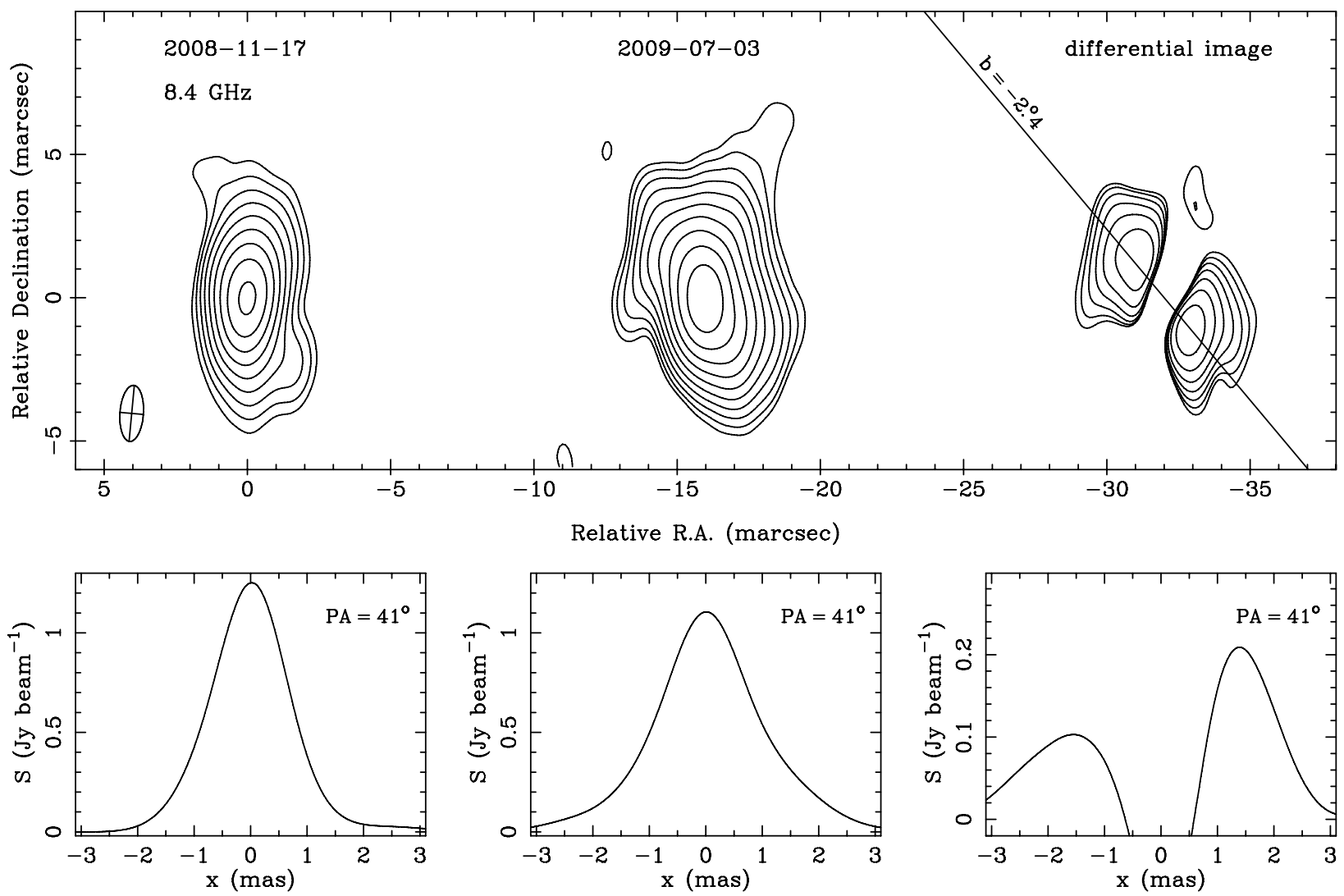

Fig. 4. Same analysis as in Fig. 3 but for different epochs and at $8.4 \mathrm{GHz}$.

To analyze the refractive-induced pattern detected on May 28, 2009 in more detail we fit the observed brightness distribution at this epoch with Gaussian emission features (Table 2) and performed difference imaging with the previous epoch, 2008 November 26, which appears to be the least affected by scattering (Fig. 3). The pattern is quasi-symmetric, and consists of a hierarchy of secondary images produced by ray crossings. The best fit model contains four Gaussian components that are progressively de-magnified with angular separation from the primary image, with two closer sub-images at the level of $\sim 10 \%$ of the core flux density and two outer ones at $\sim(3-4) \%$ level. The pattern is stretched out along the line of constant galactic latitude. A noteworthy feature of the pattern is that it exhibits sub-images on either side of the core, while in a typical ESE event these are expected to lie only on one side (Clegg et al. 1998). This could be explained by complex structure of the screen, having free-electron density enhancements separated by an angular distance comparable to the VLBI core extent, i.e. a double-lens system, like those investigated by Kim (2005). In this case, two secondary images could be created on both sides of the VLBI core, if the density profile of each lens is close to Gaussian (Clegg et al. 1998). The primary image would likely show some elongation. This is consistent with the observed ellipticity of the VLBI core component (Fig. 3, Table 2).

We did not detect any significant image wander of the type expected for refractive ESE (Clegg et al. 1998). Our observations were not carried out in a phase-reference mode, and during imaging we performed self-calibration that erases all absolute position information, making relative position shift analysis impossible. On the other hand, as the lens covered only the most compact component, the VLBA core, it did not affect the
Table 2. 15 GHz Gaussian component model at epoch 2009 May 28.

\begin{tabular}{lccccccc}
\hline \hline Comp. & $\begin{array}{c}S \\
(\mathrm{mJy})\end{array}$ & $S / N$ & $\begin{array}{c}r \\
(\mathrm{mas})\end{array}$ & $\begin{array}{c}\varphi \\
(\mathrm{deg})\end{array}$ & $\begin{array}{c}\text { Maj. } \\
(\mathrm{mas})\end{array}$ & Ratio & $\begin{array}{c}\text { PA } \\
(\mathrm{deg})\end{array}$ \\
$(1)$ & $(2)$ & $(3)$ & $(4)$ & $(5)$ & $(6)$ & $(7)$ & $(8)$ \\
\hline $\mathrm{C}$ & 3649 & 833 & $\ldots$ & $\ldots$ & 0.53 & 0.58 & 33 \\
$\mathrm{~J}$ & 51 & 18 & 0.75 & -11 & 0.28 & 1.00 & $\ldots$ \\
$\mathrm{J}$ & 121 & 17 & 1.54 & -20 & 1.13 & 1.00 & $\ldots$ \\
$\mathrm{S}$ & 393 & 166 & 0.77 & -144 & 0.29 & 1.00 & $\ldots$ \\
$\mathrm{S}$ & 302 & 70 & 0.78 & 33 & 0.38 & 1.00 & $\ldots$ \\
$\mathrm{S}$ & 144 & 83 & 1.21 & -138 & 0.27 & 1.00 & $\ldots$ \\
$\mathrm{S}$ & 119 & 28 & 1.45 & 33 & 0.45 & 1.00 & $\ldots$ \\
\hline
\end{tabular}

Notes. Columns are as follows: (1) component designation: $\mathrm{C}$ is the core, $\mathrm{J}$ is the jet, $\mathrm{S}$ is the secondary image component; (2) fitted Gaussian flux density at $15 \mathrm{GHz}$; (3) signal-to-noise ratio determined as the ratio of the peak flux density of the component to the rms noise under it; (4) position offset from the core component; (5) position angle of the component with respect to the core component; (6) FWHM major axis of the fitted Gaussian; (7) axial ratio of the fitted Gaussian; (8) major axis position angle of the fitted Gaussian.

position of the rest of the real jet structure, which we were able to use as the reference location when applying the two-dimensional cross correlation technique (Lewis 1995).

The signatures of refractive sub-images at epochs 2008 July 17, 2009 May 28, 2009 July 23, 2009 December 10, 2010 August 6, 2010 December 23, and 2011 May 21 are all preferentially aligned along the constant galactic latitude line with respect to the VLBI core (Fig. 1). This indicates that the discrete structures of electron density enhancements acting as refractive 
lenses move parallel to the Galactic plane, which corresponds to the direction from north-east to south-west on the images in the equatorial coordinates. This is consistent with the changes in the induced patterns between the epochs of May and July 2009, implying that the observed proper motion of the screen is prograde with regard to the galactic center. However, emission with a high signal-to-noise ratio was detected due east of the core at one fulltrack pre-MOJAVE epoch with high sensitivity, September 15, 2005, presumably suggesting that a lens drifted over the source along the tangent line. The remaining epochs, with the possible exception of November 26, 2008, are characterized by angular broadening of the VLBI core region. Interestingly, the secondary images that are also detected in linear polarization, e.g., on May 28, 2009, December 23, 2010, and May 21, 2011, show EVPAs aligned with those in the primary image.

\subsection{Additional evidence from $8 \mathrm{GHz}$ VLBA observations}

The quasar $2023+335$ was also observed with the VLBA at $8.4 \mathrm{GHz}$ on five occasions from November 2008 to November 2009 as a phase-reference calibrator in a framework of the black hole X-ray binary V404 Cyg project (Miller-Jones et al. 2009). We reduced these archival VLBA data and found structural behavior very similar to our $15.4 \mathrm{GHz}$ results. The source was least affected by the ISM in November 2008, but revealed multiplyimaged structure stretched out along the constant galactic latitude line at other epochs, including the 3 July 2009 epoch (Fig. 4), which was only 20 days prior to our $15.4 \mathrm{GHz}$ MOJAVE epoch. The $8.4 \mathrm{GHz}$ peaks of the induced structure are separated by $\sim 3.0$ mas, while at $15.4 \mathrm{GHz}$ the separation is $\sim 0.8$ mas on 23 July 2009 ( $\sim 1.0$ mas on 28 May 2009). The separation thus scales as $\lambda^{2.2}$ ( $\lambda^{1.75} 28$ May 2009), which we consider to be convincing proof of a plasma scattering origin for the multiple imaging event (Rickett \& Coles 1988), because the refraction angle in plasma scattering is expected to follow a $\lambda^{2}$ dependence (Clegg et al. 1998), while for the intrinsic scenario the studied parameter of the angular separation is frequency-independent since the synchrotron jet emission is optically thin (e.g., Pushkarev \& Kovalev 2012).

\subsection{Summary of observational evidence in support of refractive scattering}

To summarize, we have found several pieces of observational evidence which suggest that the multiple imaging event witnessed in 2009 in $2023+335$ was caused by refraction in the interstellar medium:

- the 8 and $15 \mathrm{GHz}$ parsec-scale jet structure shows strongly induced patterns at two epochs, with weaker manifestations of the effect seen at several other epochs;

- the strongly induced patterns are coincident with an ESE event in the radio light curve, as predicted by models;

- the source is located in the Galactic plane behind the Cygnus loop, which provides a high probability of a propagation effect through an intervening turbulent screen;

- the angular separation between the peaks of the induced structure shows a $\lambda^{2}$-dependence, which is convincing proof of a plasma scattering origin of the induced sub-images. This argument also rules out a scenario in which the observed structural changes between epoch of Nov. 2008 and May 2009 are intrinsic to the source.

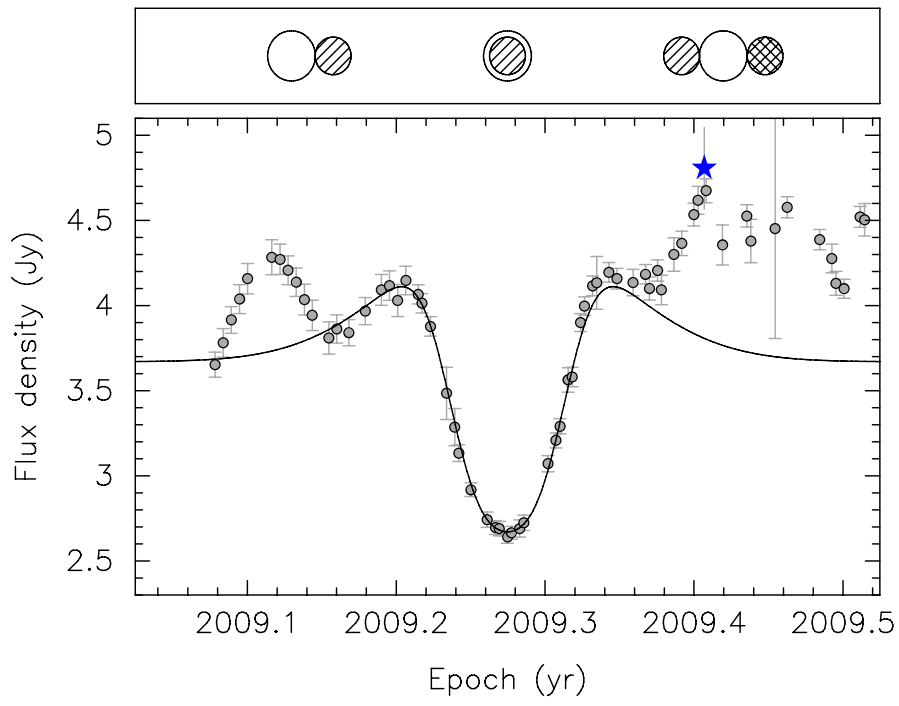

Fig. 5. Bottom: $15 \mathrm{GHz}$ OVRO light curve in the quasar 2023+335 during an extreme scattering event. The solid line shows the stochastic broadening model light curve for the ESE. A two-component model was used: a lensed component of flux density $1.87 \mathrm{Jy}$, and an un-lensed component of flux density $1.80 \mathrm{Jy}$. The star symbol represents the MOJAVE epoch that revealed multi-component structure induced by refraction (see Fig. 3). This epoch of VLBA observations serendipitously coincided with the local sharp spike, possibly ascribed to the outer caustic that is associated with the passage of the lens edge over the background source. Top: a toy model of the scattering screen during the ESE. The unfilled circle represents the $15.4 \mathrm{GHz}$ VLBA core of the background quasar. The hatched circle represents the lens that passes over the source. The cross-hatched circle represents another electron density enhancement of the screen.

\section{Derived properties of the screen}

We can draw some basic conclusions from a simple analysis of the shape of the ESE light curve (Fig. 5). The rounded minimum of the ESE suggests that the lens is comparable to or smaller in size than the part of the source which it occults; otherwise, a flatbottomed minimum is expected. Also, the ESE caused a 30\% decrease in the source's total flux density at $15 \mathrm{GHz}$. This indicates that the lensed part of the source contains a significant fraction of the total flux density. The most likely candidates for such a region are the VLBI core and/or, if present and bright, the innermost VLBI jet component, because (i) the source is highly core dominated on milliarcsecond scales and (ii) the total VLBA flux density agrees well with the single-dish flux density of the source, indicating that virtually all the $15 \mathrm{GHz}$ emission originates from milliarcsecond scales. Finally, the sharp spike at the epoch of 2009 .4 is likely attributable to an outer caustic that is associated with the passage of the lens edge over the background source. The presence of this caustic suggests that the refractive scattering is strong, and multiple images are expected to be formed (Fig. 3). The other outer caustic at the epoch of $\sim 2009.1$ is present but less pronounced, indicating a difference in the free-electron density profile across the scattering screen.

\subsection{Stochastic broadening}

To derive the quantitative parameters of the plasma lens, we used a statistical model for flux redistribution developed by Fiedler et al. (1994) and based on stochastic broadening regardless of its nature, refractive or diffractive. In this model, the flux density of a distant background source at a time $t$ during an ESE is 
determined as $f\left(t ; I_{0}, \mu, \theta_{s}, \theta_{l}, \theta_{b}\right)$, where $I_{0}$ is the nominal (unlensed) flux density level of the source outside the lens, $\mu$ is the proper motion of the lens across the line of sight, $\theta_{s}$ is the intrinsic FWHM angular size of the source, $\theta_{l}$ is the apparent angular width of the lens (which has a band-shaped geometry when projected on the sky), and all parallel rays incident on the lens are assumed to be scatter-broadened into a Gaussian brightness distribution of FWHM $\theta_{b}$. Both $\theta_{l}$ and $\theta_{b}$ are measured with respect to $\theta_{s}$; thus we fit their ratios.

We used a two-component model, with a lensed component $I_{\text {scat }}(t)$ being subject to temporal flux density variations from scattering during the ESE, and an un-lensed part $I_{\text {unscat }}$ such that their sum equals the nominal flux density level outside the ESE. We also assumed that there are no significant intrinsic flux density changes during the event. We then fitted $\theta_{l}, \theta_{b}, I_{0}, I_{\text {scat }}$, and $\mu$ by searching in parameter space and evaluating the goodness of fit using the $\chi^{2}$ statistic. We found good agreement with the depth and shape of the minimum being reproduced. In Fig. 5, we show the simulated light curve superposed on the $15 \mathrm{GHz}$ OVRO light curve for the set of best-fit parameters listed in Table 3 . The scattered component comprises $51 \%$ of the total flux density. This rules out a scenario where the lens drifts over any downstream jet component, because the brightest of them, at the latest prior epoch (2008 November 26), accounted for only about $10 \%$ of the $15 \mathrm{GHz}$ total flux density. Therefore, it is most likely that the lens passed over the VLBI core component.

The fitted parameters could be used to calculate the following physical characteristics of the plasma lens:

- Angular size $\theta_{l}=\theta_{s} / 1.03$, where the intrinsic angular size of the VLBI core $\theta_{s}=\theta_{b} / 1.88 \approx 0.28$ mas, and $\theta_{b} \approx 0.53$ mas is the FWHM of the observed VLBI core size at epoch 2009 May 28. We then obtained $\theta_{l} \approx 0.27$ mas, which is an upper limit since the lens passage would most likely occur along a chord rather than precisely along the diameter of the VLBI core. It is also worth noting that the observed size of the VLBI core component at the epoch 2008 November 26, which seems to show the source structure when least affected by scattering, is only $\sim 15 \%$ broader than the inferred intrinsic extent $\theta_{s}=0.28$ mas.

- Transverse linear size $a=\theta_{l} D$, where $D$ is the distance to the lens. The distance to the lens is uncertain, as the line of sight to $2023+335$ passes through the nearby $(D \sim 1.5 \mathrm{kpc}$, e.g., Rygl et al. 2012) Cygnus region in the Local arm (also known as the Orion or local spur), the Perseus arm $(D \sim$ $6 \mathrm{kpc}$ ), and the outer arm $(D \sim 10 \mathrm{kpc})$ (see Fig. 6). We assume that the lens is in the highly turbulent Cygnus region at the distance of $\sim 1.5 \mathrm{kpc}$, which yields $a \approx 0.4 \mathrm{AU}$.

- Proper motion $\mu=3.5 \theta_{s} / \tau \approx 6.8$ mas $\mathrm{yr}^{-1}$, where $\tau \approx$ $0.14 \mathrm{yr}$ is the duration of the ESE as measured between the peaks that surrounds the minimum of the flux density modulation.

- Transverse speed $V_{l \perp}=4.74 \mu_{[\mathrm{mas} / \mathrm{yr}]} D_{[\mathrm{kpc}]} \approx 48.7 \mathrm{~km} \mathrm{~s}^{-1}$ with respect to the observer. Taking into account the Earth's motion around the Sun, the projected speed of the screen at the middle phase of the ESE in the Sun's rest frame is about $56 \mathrm{~km} \mathrm{~s}^{-1}$.

The fitted light curve reproduces the inner caustics quite well. What the model does not reproduce are the flux density enhancements attributed to outer caustics, the earlier one of which shows an excess of $\sim 0.5$ Jy comparing to the unlensed flux density level, while the later outer caustic is characterized by nearly twice as large flux density excess, $\sim 1 \mathrm{Jy}$. The latter value is consistent with the total flux density of the induced quasi-symmetric
Table 3. Parameters of the best-fit stochastic broadening model.

\begin{tabular}{lcr}
\hline \hline Parameter & Value & Unit \\
\hline$\theta_{s} / \theta_{l}$ & 1.03 & \\
$\theta_{b} / \theta_{s}$ & 1.88 & \\
$I_{0}$ & 3.67 & $\mathrm{Jy}$ \\
$I_{\text {scat }} / I_{0}$ & 0.51 & \\
$\tau^{\mathrm{a}}$ & 0.14 & $\mathrm{yr}$ \\
$\mu$ & 25.01 & $\theta_{s} \mathrm{yr}^{-1}$ \\
$\chi^{2}$ & 0.02 & \\
\hline
\end{tabular}

Notes. ${ }^{(a)}$ This parameter held fixed.

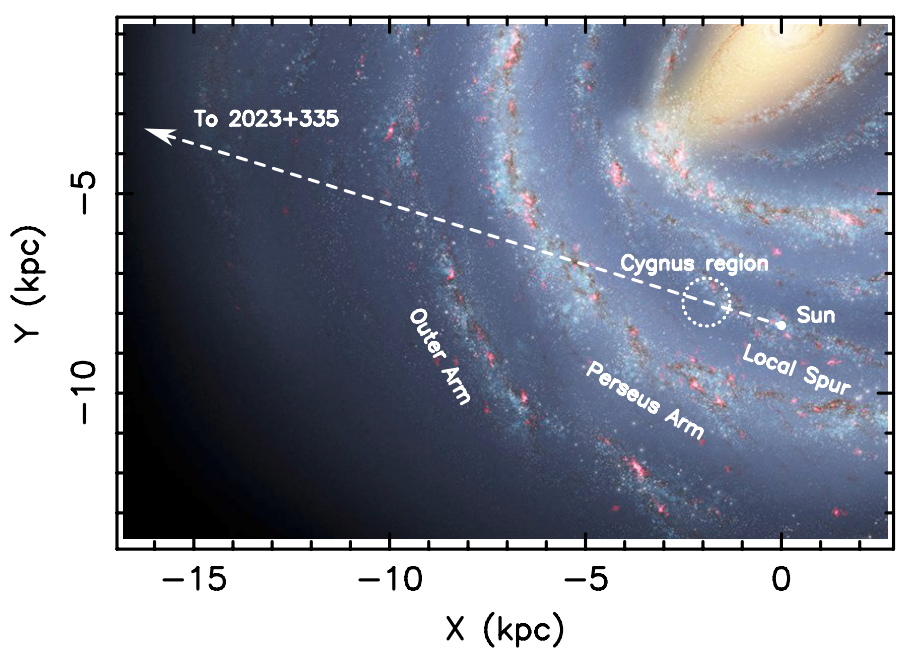

Fig. 6. Artist's impression of a face-on view of the Galactic plane (image credit: R. Hurt, NASA/JPL-Caltech/SSC). The Sun is placed at $8.3 \mathrm{kpc}$ (Gillessen et al. 2009) from the Galactic center $(0,0)$. The dashed line shows a direction toward $2023+335$ with a galactic longitude $l=73^{\circ} .13$ that passes through the nearby $(D \sim 1.5 \mathrm{kpc})$ Cygnus region in the Local arm, the Perseus arm $(D \sim 6 \mathrm{kpc})$, and the outer $\operatorname{arm}(D \sim 10 \mathrm{kpc})$. We assume that the lens is located in the turbulent Cygnus region (dotted circle) at the distance of $\sim 1.5 \mathrm{kpc}$.

multi-component pattern (see 'S' components in Table 2), indicating that the scattering screen at epoch 2009 May 28 has a double-lens structure (Fig. 5, upper panel) that creates the secondary images on both sides from the VLBI core, as depicted in Fig. 3, and thus considerably deviates from the model light curve.

\subsection{Refraction defocusing}

Another approach is provided by the refraction defocusing (RD) model (Clegg et al. 1998), in which the intervening scattering screen is represented by a plasma lens with a Gaussian profile of free-electron column density. The refractive properties of the lens in this model are described completely by an introduced dimensionless parameter

$\alpha=3.6\left(\frac{\lambda}{1 \mathrm{~cm}}\right)^{2}\left(\frac{N_{0}}{1 \mathrm{pc} \mathrm{cm}^{-3}}\right)\left(\frac{D}{1 \mathrm{kpc}}\right)\left(\frac{a}{1 \mathrm{AU}}\right)^{-2}$,

where $N_{0}$ is the maximum column density through the lens and $\lambda$ is the observing wavelength. In case of moderate and strong refraction, the caustic spikes are expected to be formed. The relative separation between the inner $\Delta x_{i}^{\prime}$ and outer $\Delta x_{o}^{\prime}$ pair of 
caustics is an important observable that allows us to place a constraint on $\alpha$ through the following analytic expression (Clegg et al. 1998, Eq. (23))

$\alpha \simeq 1.7\left(3 \frac{\Delta x_{o}^{\prime}}{\Delta x_{i}^{\prime}}-1\right)^{1.06}$.

For the ESE in $2023+335$, the ratio $\Delta x_{o}^{\prime} / \Delta x_{i}^{\prime} \approx 2$. Then the strength of the lens is $\alpha \simeq 10$. We can now derive the following properties of the lens:

- maximum electron column density through the lens $N_{0}=$ $0.28 \alpha \lambda_{[\mathrm{cm}]}^{-2} D_{[\mathrm{kpc}]} \theta_{l[\mathrm{mas}]}^{2} \approx 2.5 \times 10^{17} \mathrm{~cm}^{-2}$, where $\lambda$ is the wavelength of observation in $\mathrm{cm}$;

- free-electron density within the lens $n_{\mathrm{e}} \approx N_{0} / a \approx 4.0 \times$ $10^{4} \mathrm{~cm}^{-3}$;

- mass of the lens $M_{1} \sim m_{\mathrm{p}} n_{\mathrm{e}} a^{3}$, where $m_{\mathrm{p}}$ is the proton mass. For a lens at $1.5 \mathrm{kpc}, M_{1} \sim 1.4 \times 10^{19} \mathrm{~g} \sim 7.2 \times 10^{-15} M_{\odot}$.

It is worth noting that the inner caustics are less pronounced in comparison with the outer one clearly seen at the epoch of 2009.4 in Fig. 5, while in the RD model they are expected to have comparable levels. However, simulations performed by Stinebring et al. (2007) considering a two-dimensional (rather than a one-dimensional as in the RD model) lens with a Gaussian density profile have shown the absence of strong inner caustics. More complex cases of double-lens systems have been investigated by Kim (2005).

\section{Turbulent angular broadening}

Diffraction phenomena associated with scattering by a turbulent interstellar medium results in the angular broadening of a distant background radio source. Thus, its observed brightness distribution is a convolution of the intrinsic source structure with a scattering function. The angular size of a broadened source scales with frequency through the following relation (Rickett 1977)

$\theta_{\text {scat }} \propto v^{k}, \quad k=-\frac{\beta}{\beta-2}$

where $\beta$ is the index of the assumed power-law spectrum of the turbulent electron density fluctuations $\delta n_{\mathrm{e}} / n_{\mathrm{e}}$ (Cordes et al. 1985)

$P_{\delta n_{\mathrm{e}}}(q)=C_{n}^{2} q^{-\beta}, \quad q_{0} \leq q \leq q_{1}$,

where $C_{n}^{2}$ is a normalizing constant, and $q_{0}$ and $q_{1}$ are spatial wave-number cutoffs corresponding to the outer and inner scales of the turbulence, respectively, bracketing over 6 orders of magnitude of irregularity scales from $\sim 10^{8} \mathrm{~cm}$ to $\sim 10^{15} \mathrm{~cm}$ (Armstrong et al. 1995; Combes 2000; Cordes \& Wolszczan 1986). A theory of turbulence developed by Kolmogorov (1941) predicts that $\beta=11 / 3$ for isotropic density fluctuations, and it has been reported that observational data are consistent with this value for a number of lines of sight (Armstrong et al. 1981; Wolszczan 1983; Rickett 1990; Fey et al. 1991). In this case, the scattered angular size is expected to be proportional to $v^{-2.2}$. However, the theory accommodates steeper density fluctuation spectra $(\beta>4)$ as well (Cordes \& Lazio 2001), being observed for some other lines of sight (Hewish et al. 1985; Romani et al. 1986). Scattering can manifest itself through diffractive and/or refractive effects, depending on the size of turbulent eddies and associated turbulence spectrum. Diffractive effects will dominate for a screen with small-scale electron-density irregularities, yielding shallow $(\beta<4)$ spectra, while considerably
Table 4. VLBI core parameters at different observing frequencies/epochs.

\begin{tabular}{lccc}
\hline \hline $\begin{array}{l}\text { Frequency } \\
(\mathrm{GHz})\end{array}$ & $\begin{array}{c}\text { Epoch } \\
\text { (yyy-mm-dd) }\end{array}$ & $\begin{array}{c}\text { Flux density } \\
(\mathrm{Jy})\end{array}$ & $\begin{array}{c}\text { Angular size } \\
\text { (mas) }\end{array}$ \\
\hline 1.421 & $1995-06-23$ & $1.08 \pm 0.28$ & $28.49 \pm 7.89$ \\
2.265 & $1995-10-12$ & $1.93 \pm 0.44$ & $11.89 \pm 2.99$ \\
2.269 & $1996-05-15$ & $1.94 \pm 0.47$ & $11.08 \pm 2.99$ \\
2.292 & $2001-03-12$ & $1.20 \pm 0.37$ & $9.65 \pm 3.13$ \\
2.309 & $2003-07-09$ & $0.73 \pm 0.36$ & $14.78 \pm 3.75$ \\
8.335 & $1995-10-12$ & $2.66 \pm 0.41$ & $0.95 \pm 0.21$ \\
8.339 & $1996-05-15$ & $2.62 \pm 0.42$ & $0.74 \pm 0.20$ \\
8.646 & $2001-03-12$ & $1.79 \pm 0.34$ & $1.00 \pm 0.23$ \\
8.646 & $2003-07-09$ & $1.05 \pm 0.26$ & $1.25 \pm 0.37$ \\
8.322 & $2010-01-07$ & $3.17 \pm 0.50$ & $1.23 \pm 0.26$ \\
15.365 & $2000-04-02$ & $0.89 \pm 0.09$ & $0.56 \pm 0.08$ \\
15.365 & $2001-09-19$ & $1.54 \pm 0.16$ & $0.39 \pm 0.07$ \\
15.365 & $2002-02-15$ & $1.27 \pm 0.11$ & $0.32 \pm 0.06$ \\
15.365 & $2008-07-17$ & $1.27 \pm 0.07$ & $0.46 \pm 0.04$ \\
15.357 & $2008-11-26$ & $2.80 \pm 0.15$ & $0.36 \pm 0.03$ \\
15.357 & $2009-05-28$ & $3.65 \pm 0.15$ & $0.53 \pm 0.03$ \\
15.357 & $2009-07-23$ & $3.44 \pm 0.15$ & $0.48 \pm 0.03$ \\
15.357 & $2009-10-27$ & $1.87 \pm 0.10$ & $0.37 \pm 0.04$ \\
15.357 & $2009-12-10$ & $4.87 \pm 0.21$ & $0.35 \pm 0.03$ \\
15.357 & $2010-02-11$ & $4.52 \pm 0.15$ & $0.48 \pm 0.02$ \\
15.357 & $2010-08-06$ & $4.30 \pm 0.18$ & $0.35 \pm 0.03$ \\
15.357 & $2010-10-25$ & $2.05 \pm 0.13$ & $0.41 \pm 0.04$ \\
15.357 & $2010-12-24$ & $2.16 \pm 0.07$ & $0.45 \pm 0.02$ \\
15.357 & $2011-05-21$ & $1.08 \pm 0.04$ & $0.40 \pm 0.02$ \\
15.357 & $2011-09-12$ & $1.32 \pm 0.06$ & $0.39 \pm 0.03$ \\
15.357 & $2012-07-12$ & $2.51 \pm 0.14$ & $0.45 \pm 0.04$ \\
15.357 & $2012-11-11$ & $2.31 \pm 0.19$ & $0.38 \pm 0.05$ \\
22.204 & $2008-06-01$ & $1.39 \pm 0.16$ & $0.24 \pm 0.05$ \\
22.241 & $2010-04-24$ & $4.71 \pm 0.28$ & $0.23 \pm 0.03$ \\
22.240 & $2010-07-26$ & $2.97 \pm 0.27$ & $0.27 \pm 0.04$ \\
22.239 & $2010-09-25$ & $3.03 \pm 0.32$ & $0.24 \pm 0.05$ \\
22.238 & $2010-11-18$ & $2.41 \pm 0.15$ & $0.28 \pm 0.03$ \\
22.238 & $2010-12-16$ & $1.37 \pm 0.12$ & $0.26 \pm 0.04$ \\
22.241 & $2011-04-25$ & $1.64 \pm 0.13$ & $0.28 \pm 0.04$ \\
24.433 & $2006-06-04$ & $2.05 \pm 0.15$ & $0.21 \pm 0.03$ \\
86.248 & $2002-04-20$ & $0.42 \pm 0.12$ & $0.03 \pm 0.02$ \\
\hline & & & \\
& & & \\
& 257 & &
\end{tabular}

Notes. Columns are as follows: (1) central observing frequency; (2) epoch of observations; (3) fitted Gaussian flux density; (4) FWHM angular size of the fitted Gaussian.

more refraction is expected for large-scale turbulent eddies causing steep $(\beta>4)$ spectra (Cordes \& Wolszczan 1986). Note that if no scattering is present, the observed angular size of a flat-spectrum, inhomogeneous synchrotron source is expected to scale approximately as $v^{-1}$ (Kellermann \& Pauliny-Toth 1981), although departures from this dependence are also possible and can be caused by pressure and density gradients in the jet or by external absorption from the surrounding medium (Lobanov 1998; Kovalev et al. 2008; Sokolovsky et al. 2011; Pushkarev et al. 2012, and references therein).

To investigate the amount and nature of scattering occurring along the line of sight to $2023+335$, we used the $15 \mathrm{GHz}$ MOJAVE data together with other publicly available ${ }^{2}$ VLBI data for $2023+335$ at other frequencies, such as $1.4,2,8,15,24$, and $86 \mathrm{GHz}$ (see Table 4). The archival data for all experiments except two, carried out in Oct 1995 at 2 and $8 \mathrm{GHz}$ (Fey \& Charlot 1997) and Apr 2002 at 86 GHz (Lee et al. 2008), were reduced by us. The source structure was model fitted with circular Gaussian components that after being convolved with the

2 http://astrogeo.org/vlbi_images 


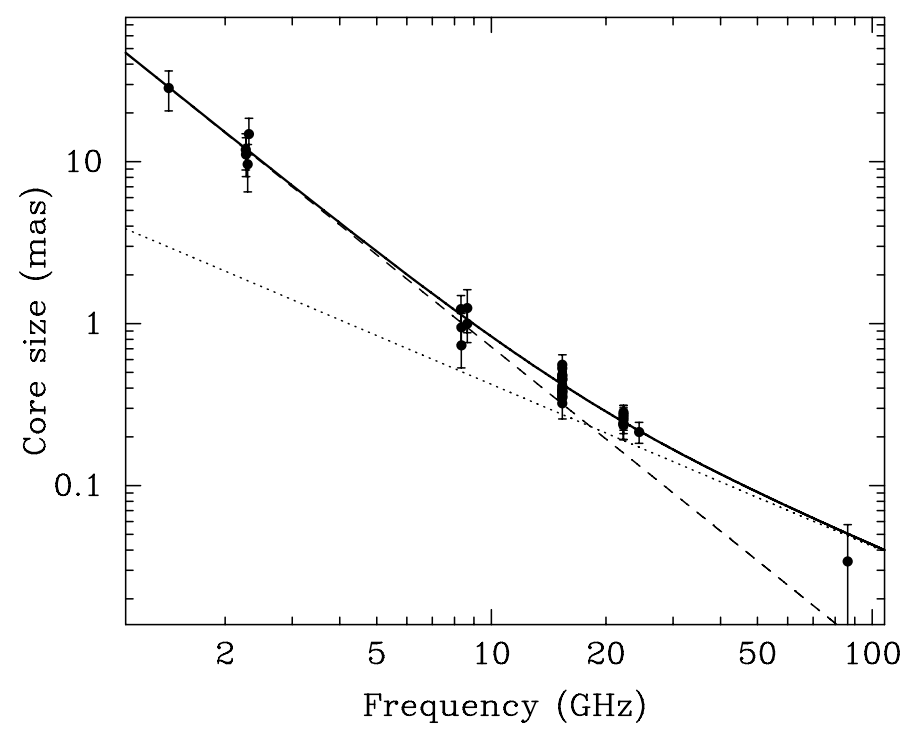

Fig. 7. Observed angular size of the VLBI core component as a function of observing frequency using all available VLBI data. The solid line represents the fit of Eq. (5), the dashed line indicates the inferred scattering diameter, and the dotted line shows the inferred intrinsic diameter.

restoring beam, adequately reproduce the constructed brightness distribution. In Fig. 7, we plot the angular size of the VLBI core component as a function of observing frequency listed in Table 4. We fitted the measured angular width to the following relation (Lazio et al. 2008)

$\theta_{\text {observed }}^{2}=\left(\theta_{\text {scat }} v^{k}\right)^{2}+\left(\theta_{\text {int }} v^{-1}\right)^{2}$

where $\theta_{\text {scat }}$ and $\theta_{\text {int }}$ are the scattered and intrinsic FWHM diameters of the VLBI core, respectively, scaled to a frequency of $1 \mathrm{GHz}$. We used a grid-search method to fit for $\theta_{\text {scat }}, \theta_{\text {int }}$, and $k$ that produced the minimum $\chi^{2}$. We found the following bestfitting values $\theta_{\text {scat }}=55.8$ mas, $\theta_{\text {int }}=4.2 \mathrm{mas}$, and the index $k=-1.89$, which corresponds to $\beta=4.3$. This indicates that the angular size of the VLBI core increases faster than would be expected if there is no scattering, but not as fast as in case of scattering caused by a Kolmogorov electron density spectrum.

Partly, this can be due to the non-simultaneity of the VLBI observations, given the maximum epoch difference of $\sim 16 \mathrm{yr}$ between the $2 / 8 \mathrm{GHz}$ and $15 \mathrm{GHz}$ data. On the other hand, the observations at 2 and $8 \mathrm{GHz}$ at four epochs were carried out simultaneously, thereby providing more robust estimates of the $k$-index, although determined within a $\sim 10$ times narrower frequency range, with the average value of $-1.90 \pm 0.13(\beta \approx 4.2)$. This is consistent with the one calculated from the all nonsimultaneous data covering a broader frequency range. This average two-frequency value of $k$ also agrees well with the power index $k=-1.97 \pm 0.29$ derived by Fey et al. (1989b) from earlier non-simultaneous VLBI observations of $2023+335$ performed at $0.61,1.66$, and $4.99 \mathrm{GHz}$, but still deviates from the conventional index of -2.2 expected for a Kolmogorov spectrum. The following questions then arise: (i) is it a result of a non-Kolmogorov turbulence, e.g., caused by anisotropy effects?; (ii) what may cause the weaker frequency dependence?; and (iii) why the slope of Fey et al. (1989b) (-1.97) is closer to that of Kolmogorov $(-2.2)$ than our estimate of the slope $(-1.89)$ ? The currently most accepted model of magnetohydrodynamic turbulence developed by Goldreich \& Sridhar (1995), in which the compressible regime was tested numerically (Cho \& Lazarian 2002, 2003), includes anisotropy effects and predicts the Kolmogorov spectrum. As for the second question, Cordes \& Lazio (2001) reported that the scaling law of angular size with frequency can be considerably shallower than the canonical $v^{-11 / 5}$ dependence if the scattering screen with Kolmogorov fluctuations has a confined structure or is truncated transverse to the line of sight, i.e. the "container" in which the turbulence resides has boundaries. This is consistent with the observed ESE that likely indicates the presence of discrete density structures, the observational manifestations of which we discussed in Sect. 3. To speculate on the third question, we see two possible explanations: (1) the data used by Fey et al. (1989b) probed somewhat different (larger) spatial scales, because the lowest frequency in their data was $0.61 \mathrm{GHz}$, while that in our set of data is higher by a factor of $2.3,1.4 \mathrm{GHz}$; (2) the properties of the screen, as a function of time, could be different at epoch of 1985-1986 studied by Fey et al. (1989b). Most probably, both of these possibilities play a role to some degree.

The most prominent angular broadening occurs at the lowest of the available observing frequencies, $1.4 \mathrm{GHz}$, and increases the angular size by a factor of $\sim 10$ as seen in Fig. 7. The expected size of the core component, if there were no screen, is within typical VLBI core sizes of $\sim(1-2)$ mas at $2 \mathrm{GHz}$ and $\sim(0.3-0.6)$ mas at $8 \mathrm{GHz}$ as derived from a sample of 370 sources (Pushkarev \& Kovalev 2012), and $\sim(0.2-0.4)$ mas at $15 \mathrm{GHz}$ from a sample of 133 sources (Kovalev et al. 2005).

From the above analysis, we can constrain the turbulence spectrum index toward $2023+335$ to within the range $4.2 \lesssim \beta \lesssim$ 4.7. We note that such steep turbulence spectra $(\beta \sim 4.3)$ have been seen previously in nature, specifically in laboratory scattered laser light experiments (Jakeman \& Jefferson 1984; Walker \& Jakeman 1984), suggesting that spectra that are steeper than the Kolmogorov spectrum may not be that unusual.

\section{Summary}

We have found convincing evidence for the first detected multiple imaging of an AGN jet due to refractive foreground scattering in our galaxy. This rare phenomenon was theoretically predicted several decades ago and is based on the refractive properties of localized electron density enhancements in the ionized component of the Galactic interstellar medium (Lovelace 1970; Cordes et al. 1986; Rickett \& Coles 1988). We detected the effect in the low galactic latitude $\left(b=-2^{\circ} .4\right)$ quasar $2023+335$ in a number of VLBA observations obtained between 2008 and 2012 as part of the MOJAVE program. Its strongest manifestation, at $\sim 10 \%$ of the primary image flux density, occurred on May 28, 2009 at $15 \mathrm{GHz}$, when the source was undergoing an extreme scattering event, specifically during a special phase when a caustic spike associated with the lens edge passed over the source. We observed a highly significant multi-component pattern of secondary images induced by strong refraction in May 2009 and July 2009, which was stretched out roughly along the constant galactic latitude line with $\mathrm{PA} \approx 40^{\circ}$. This suggests that the direction of relative motion of the lens is parallel to the galactic plane, as expected for an orbiting cloud. We observed sporadic weaker but still detectable patterns at similar position angles with respect to the VLBI core at other epochs. Using archival VLBA data, we were able to detect the same effect at a lower observing frequency, $8.4 \mathrm{GHz}$. The angular separation of the peaks of the scatter-induced structure follows a $\lambda^{2}$ dependence, which provides strong evidence for a plasma scattering origin of the multiple imaging. The parsec-scale source structure, when unaffected by scattering, consists of a bright core 
that typically accounts for $\sim 80 \%$ of the total VLBA flux density, and a short jet extending along $\mathrm{PA} \approx-20^{\circ}$. Our multiple imaging observations provide valuable information for discriminating between various competing models of ESE, the true nature of which is still uncertain.

By taking into account the length of the ESE event ( $~ 0.14 \mathrm{yr})$ and by assuming that it was caused by an ionized gas cloud drifting across the line of sight, we determined the proper motion of the lens: $\sim 6.8$ mas $\mathrm{yr}^{-1}$, and its angular size: $\sim 0.27$ mas. The latter is comparable to the intrinsic size of the VLBI core at $15 \mathrm{GHz}: \sim 0.28$ mas $(\sim 1 \mathrm{pc})$. The line of sight toward $2023+335$ passes through the highly turbulent Cygnus region located at a distance of about $1.5 \mathrm{kpc}$. Assuming that that the lens lies at this distance, its transverse speed and linear size are $\sim 56 \mathrm{~km} \mathrm{~s}^{-1}$ and $\sim 0.4$ AU, respectively.

Our observations support a model of ESE suggested by Clegg et al. (1998), since formation of multiple imaging of the background source along with formation of caustics surfaces in a light curve predicted by the model have been detected. However, we were not able to detect any substantial angular position wander of the background source, as predicted by the model.

Analyzing the non-simultaneous multi-frequency VLBI observations covering a frequency range from 1.4 to $86 \mathrm{GHz}$, we found that the scattered angular size of the VLBI core scales as $v^{-1.89}$, implying the presence of a highly turbulent, refractive dominated scattering screen along the line of sight to $2023+335$. The shallower than canonical Kolmogorov $v^{-2.2}$ dependence may be caused by a confined structure of the screen with arbitrary spatial variations of scattering power in the transverse direction.

We note that future multiple imaging events in AGN caused by refraction scattering in the interstellar medium can be successfully detected if the following two conditions are fulfilled: (i) a target is observed by a multi-band VLBI when it is passing through the caustic surface of an ESE; (ii) the refractive power of the lens is sufficiently strong to separate the secondary images at angular distances larger than the scattered diameter of the primary image.

Acknowledgements. The authors acknowledge K.I. Kellermann, E. ClausenBrown, and the other members of the MOJAVE team. We are also grateful to J. Cordes, B. Rickett and T. J. W. Lazio for productive discussions. We thank the anonymous referee for useful comments which helped to improve the manuscript. This research has made use of data from the MOJAVE database that is maintained by the MOJAVE team (Lister et al. 2009a). The MOJAVE project is supported under NASA Fermi grant 11-Fermi11-0019. Part of this work was supported by the COST Action MP0905 "Black Holes in a Violent Universe". A.B.P. was partially supported by DAAD and the "Nonstationary processes in the Universe" Program of the Presidium of the Russian Academy of Sciences. Y.Y.K. was supported by the Russian Foundation for Basic Research (projects 11-02-00368, 12-02-33101), the basic research program "Active processes in galactic and extragalactic objects" of the Physical Sciences Division of the Russian Academy of Sciences, and the Dynasty Foundation. T.H. was supported by the Jenny and Antti Wihuri foundation. E.R. was partially supported by the Spanish MINECO projects AYA2009-13036C02-02 and AYA2012-38491-C02-01 and by the Generalitat Valenciana project PROMETEO/2009/104. The UMRAO monitoring program is supported in part by NASA Fermi GI grants NNX09AU16G, NNX10AP16G and NNX11AO13G and NSF grant AST-0607523. The OVRO 40-m monitoring program is supported in part by NASA grants NNX08AW31G and NNX11A043G, and NSF grants AST-0808050 and AST-1109911. The VLBA is a facility of the National Science Foundation operated by the National Radio Astronomy Observatory under cooperative agreement with Associated Universities, Inc. This research has made use of BM046, RDV26,40, BM290A-E, BR149, BM167, BM231, GM064, BR145D experiment data from the public VLBA archive of correlated data. This work made use of the Swinburne University of Technology software correlator (Deller et al. 2011), developed as part of the Australian Major National Research Facilities Programme and operated under licence.

\section{References}

Aller, H. D., Aller, M. F., Latimer, G. E., \& Hodge, P. E. 1985, ApJS, 59, 513 Armstrong, J. W., Cordes, J. M., \& Rickett, B. J. 1981, Nature, 291, 561 Armstrong, J. W., Rickett, B. J., \& Spangler, S. R. 1995, ApJ, 443, 209 Baars, J. W. M., Genzel, R., Pauliny-Toth, I. I. K., \& Witzel, A. 1977, A\&A, 61, 99

Blandford, R. D., \& Königl, A. 1979, ApJ, 232, 34

Bochkarev, N. G., \& Sitnik, T. G. 1985, Ap\&SS, 108, 237

Boettcher, M. 2010 [arXiv : 1006. 5048]

Chepurnov, A., \& Lazarian, A. 2010, ApJ, 710, 853

Cho, J., \& Lazarian, A. 2002, in BAAS, 34, 1124

Cho, J., \& Lazarian, A. 2003, in Rev. Mex. Astron. Astrofis. Conf. Ser. 15, eds. J. Arthur, \& W. J. Henney, 293

Cimò, G., Beckert, T., Krichbaum, T. P., et al. 2002, PASA, 19, 10

Clegg, A. W., Chernoff, D. F., \& Cordes, J. M. 1988, in Radio Wave Scattering in the Interstellar Medium, ed. J. M. Cordes, B. J. Rickett, \& D. C. Backer, AIP Conf. Ser., 174, 174

Clegg, A. W., Fey, A. L., \& Lazio, T. J. W. 1998, ApJ, 496, 253

Combes, F. 2000, Adv. Ser. Astrophys. Cosmol., 10, 143

Cordes, J. M., \& Lazio, T. J. W. 2001, ApJ, 549, 997

Cordes, J. M., \& Wolszczan, A. 1986, ApJ, 307, L27

Cordes, J. M., Weisberg, J. M., \& Boriakoff, V. 1985, ApJ, 288, 221

Cordes, J. M., Pidwerbetsky, A., \& Lovelace, R. V. E. 1986, ApJ, 310, 737

Deller, A. T., Brisken, W. F., Phillips, C. J., et al. 2011, PASP, 123, 275

Dennett-Thorpe, J., \& de Bruyn, A. G. 2002, Nature, 415, 57

Dermer, C. D., Finke, J. D., Krug, H., \& Böttcher, M. 2009, ApJ, 692, 32

Elmegreen, B. G., \& Scalo, J. 2004, ARA\&A, 42, 211

Fey, A. L., \& Charlot, P. 1997, ApJS, 111, 95

Fey, A. L., Spangler, S. R., \& Mutel, R. L. 1989a, ApJ, 337, 730

Fey, A. L., Spangler, S. R., \& Mutel, R. L. 1989b, ApJ, 337, 730

Fey, A. L., Spangler, S. R., \& Cordes, J. M. 1991, ApJ, 372, 132

Fiedler, R. L., Dennison, B., Johnston, K. J., \& Hewish, A. 1987, Nature, 326, 675

Fiedler, R., Dennison, B., Johnston, K. J., Waltman, E. B., \& Simon, R. S. 1994, ApJ, 430, 581

Gillessen, S., Eisenhauer, F., Trippe, S., et al. 2009, ApJ, 692, 1075

Goldreich, P., \& Sridhar, S. 1995, ApJ, 438, 763

Goodman, J., \& Narayan, R. 1985, MNRAS, 214, 519

Greisen, E. W. 2003, in Astrophys. Information Handling in Astronomy Historical Vistas, ed. A. Heck (Dordrecht: Kluwer), Space Sci. Lib. 285, 109 Heiles, C. 1997, ApJ, 481, 193

Hewish, A., Wolszczan, A., \& Graham, D. A. 1985, MNRAS, 213, 167

Högbom, J. A. 1974, A\&AS, 15, 417

Hovatta, T., Lister, M. L., Aller, M. F., et al. 2012, AJ, 144, 105

Jakeman, E., \& Jefferson, J. H. 1984, Optica Acta, 31, 853

Jauncey, D. L., Kedziora-Chudczer, L. L., Lovell, J. E. J., et al. 2000, in Astrophysical Phenomena Revealed by Space VLBI, eds. H. Hirabayashi, P. G. Edwards, \& D. W. Murphy, 147

Jenkins, E. B., \& Tripp, T. M. 2007, in SINS - Small Ionized and Neutral Structures in the Diffuse Interstellar Medium, eds. M. Haverkorn \& W. M. Goss, ASP Conf. Ser., 365, 51

Jennison, R. C. 1958, MNRAS, 118, 276

Kara, E., Errando, M., Max-Moerbeck, W., et al. 2012, ApJ, 746, 159

Kellermann, K. I., \& Pauliny-Toth, I. I. K. 1981, ARA\&A, 19, 373

Kellermann, K. I., Vermeulen, R. C., Zensus, J. A., \& Cohen, M. H. 1998, AJ, 115,1295

Kim, H. I. 2005, Nuovo Cimento B Serie, 120, 1055

Kolmogorov, A. 1941, Akademiia Nauk SSSR Doklady, 30, 301

Komatsu, E., Dunkley, J., Nolta, M. R., et al. 2009, ApJS, 180, 330

Kovalev, Y. Y., Kellermann, K. I., Lister, M. L., et al. 2005, AJ, 130, 2473

Kovalev, Y. Y., Lobanov, A. P., Pushkarev, A. B., \& Zensus, J. A. 2008, A\&A, 483, 759

Kovalev, Y. Y., Aller, H. D., Aller, M. F., et al. 2009, ApJ, 696, L17

Kulkarni, S. R., \& Heiles, C. 1987, in Interstellar Processes, eds. D. J. Hollenbach, \& H. A. Thronson, Jr., Astrophys. Space Sci. Lib., 134, 87 Lazarian, A., \& Beresnyak, A. 2006, MNRAS, 373, 1195

Lazio, T. J. W., Fey, A. L., Dennison, B., et al. 2000, ApJ, 534, 706

Lazio, T. J. W., Gaume, R. A., Claussen, M. J., et al. 2001, ApJ, 546, 267

Lazio, T. J. W., Cordes, J. M., de Bruyn, A. G., \& Macquart, J.-P. 2004, New Astron. Rev., 48, 1439

Lazio, T. J. W., Ojha, R., Fey, A. L., et al. 2008, ApJ, 672, 115

Lee, S.-S., Lobanov, A. P., Krichbaum, T. P., et al. 2008, AJ, 136, 159

Lewis, J. P. 1995, Vision Interface, 120

Lister, M. L., \& Homan, D. C. 2005, AJ, 130, 1389

Lister, M. L., Aller, H. D., Aller, M. F., et al. 2009a, AJ, 137, 3718

Lister, M. L., Cohen, M. H., Homan, D. C., et al. 2009b, AJ, 138, 1874 
A. B. Pushkarev et al.: Refractive multiple imaging of the quasar $2023+335$

Lobanov, A. P. 1998, A\&A, 330, 79

Lovelace, R. V. E. 1970, Ph.D. Thesis, Thesis Fac. Graduate School Cornell Univ., Ithaka, N.Y.

Lovell, J. E. J., Rickett, B. J., Macquart, J.-P., et al. 2008, ApJ, 689, 108 Miller-Jones, J. C. A., Jonker, P. G., Dhawan, V., et al. 2009, ApJ, 706, L230 Pineault, S., \& Chastenay, P. 1990, MNRAS, 246, 169

Pushkarev, A. B., \& Kovalev, Y. Y. 2012, A\&A, 544, A34

Pushkarev, A. B., Kovalev, Y. Y., \& Lister, M. L. 2010, ApJ, 722, L7

Pushkarev, A. B., Hovatta, T., Kovalev, Y. Y., et al. 2012, A\&A, 545, A113

Quirrenbach, A., Witzel, A., Krichbaum, T., Hummel, C. A., \& Alberdi, A. 1989, Nature, 337, 442

Richards, J. L., Max-Moerbeck, W., Pavlidou, V., et al. 2011, ApJS, 194, 29

Rickett, B. J. 1977, ARA\&A, 15, 479

Rickett, B. J. 1990, ARA\&A, 28, 561

Rickett, B. J., \& Coles, W. A. 1988, in The Impact of VLBI on Astrophysics and

Geophysics, eds. M. J. Reid, \& J. M. Moran, IAU Symp., 129, 287

Romani, R. W., Narayan, R., \& Blandford, R. 1986, MNRAS, 220, 19

Romani, R. W., Blandford, R. D., \& Cordes, J. M. 1987, Nature, 328, 324
Rygl, K. L. J., Brunthaler, A., Sanna, A., et al. 2012, A\&A, 539, A79

Savolainen, T., \& Kovalev, Y. Y. 2008, A\&A, 489, L33

Senkbeil, C. E., Ellingsen, S. P., Lovell, J. E. J., et al. 2008, ApJ, 672, L95

Sguera, V., Malizia, A., Bassani, L., Stephen, J. B., \& Di Cocco, G. 2004, A\&A, 414,839

Shepherd, M. C. 1997, in Astronomical Data Analysis Software and Systems VI eds. G. Hunt, \& H. E. Payne (San Francisco: ASP), ASP Conf. Ser., 125, 77 Sokolovsky, K. V., Kovalev, Y. Y., Pushkarev, A. B., \& Lobanov, A. P. 2011, A\&A, 532, A38

Sowards-Emmerd, D., Romani, R. W., \& Michelson, P. F. 2003, ApJ, 590, 109 Stinebring, D., Matters, J., \& Hemberger, D. 2007, in SINS - Small Ionized and Neutral Structures in the Diffuse Interstellar Medium, eds. M. Haverkorn, \& W. M. Goss, ASP Conf. Ser., 365, 275

Twiss, R. Q., Carter, A. W. L., \& Little, A. G. 1960, The Observatory, 80, 153

Walker, J. G., \& Jakeman, E. 1984, Optica Acta, 31, 1185

Walker, M., \& Wardle, M. 1998, ApJ, 498, L125

Wolszczan, A. 1983, MNRAS, 204, 591

Zensus, J. A., Ros, E., Kellermann, K. I., et al. 2002, AJ, 124, 662 